\title{
Quick Recovery and No Arthrofibrosis in Acute Anterior Cruciate Ligament Reconstruction: a Prospective Trial of Early versus Delayed Reconstruction
}

\author{
E. Monaco ${ }^{1}$, F. Iannotti ${ }^{1}$, A. Carrozzo ${ }^{1}$, A. Annibaldi' ${ }^{1}$, F. Marzilli', M. Nurzia' ${ }^{1}$, \\ P. F. Indelli ${ }^{2}$, A. Ferretti ${ }^{1}$ \\ 1 Department of Orthopaedics, Sant'Andrea Hospital, Sapienza University of Rome, Rome, Italy \\ 2 Department of Orthopaedic Surgery and Bioengineering, Stanford University, U.S.A.
}

\section{CORRESPONDING AUTHOR:}

\section{Edoardo Monaco}

Department of Orthopaedics

Sant'Andrea Hospital

Sapienza University of Rome

via di Grottarossa 1035

00189 Rome, Italy

E-mail: edoardomonaco76@gmail.com

DOI:

10.32098/mltj.02.2021.16

LEVEL OF EVIDENCE: 4

\begin{abstract}
SUMMARY
Background. Anterior cruciate ligaments tears is one of the most frequent orthopaedics and sports medicine injuries in the athletically active population and timing of reconstruction represents a debated topic. The aim of the study is to compare range of motion (ROM) recovery and clinical outcomes between patients operated for acute reconstruction (maximum 2 weeks injury-surgery interval) and delayed reconstruction (minimum 3 weeks injury-surgery interval).

Methods. A total of 52 patients were prospectively involved in the study. 26 patients underwent acute reconstruction and 26 delayed reconstruction. A standard physical examination with Lachman and Pivot shift test and a passive ROM measurement with a goniometer were performed at each follow-up (2, 4, 8, 12 and 24 weeks postoperatively). Clinical outcomes were measured at final follow-up using Knee Injury and Osteoarthritis outcome score (KOOS), Tegner Lysholm Score and International Knee Documentation Committe (IKDC 2000) and KT-1000 evaluation. Single-leg hop test and thigh circumference measurement were performed at final follow-up.

Results. Both groups showed no statistically significant differences regarding the ROM. Full ROM was achieved 12 weeks after surgery in both groups.

The mean IKDC was 98.7 and 95.2; the mean Tegner Lysholm was 100 and 93.8 and the mean KOOS was 99 and 95.5 in the acute group and delayed ACLR group respectively. Conclusions. There were no differences between acute and delayed anterior cruciate ligament reconstruction regarding the risk of arthrofibrosis and clinical outcomes. Acute reconstruction can be performed safely with no increased risk of arthrofibrosis.
\end{abstract}

KEY WORDS

$A C L$ reconstruction; arthrofibrosis; knee function, knee; $R O M$.

\section{INTRODUCTION}

The annual anterior cruciate ligament (ACL) injury incidence reported rates for professional athletes ranges from $0.15 \%$ to $3.67 \%$ and it is higher than national population rates (median rate of $0.03 \%$ ) (1).

Although ACL reconstruction (ACLR) is considered a safe and effective procedure regardless of age (2), most reconstructions are performed in young active athletes or in patients with physically demanding occupational pursuits who are keen to resume sports or work activities as soon as possible (3).

Early surgery and rehabilitation were considered as important prerequisites for a quick recovery, however, the timing of reconstruction is a debated topic (3). Historically, delayed ACLR has been recommended since some studies reported a risk of arthrofibrosis and suboptimal clinical outcomes associated with early surgery, accordingly ACLR is often delayed recovering range of motion (ROM) and muscle strength through a preoperative rehabilitation protocol $(4,5)$. 
Recent studies, otherwise, reported no risk of arthrofibrosis with acute reconstruction showing similar or better outcomes than delayed ACLR $(6,7)$. Furthermore, early surgery seems to be more cost-effective and can decrease the risk of subsequent meniscal or cartilage lesions by reducing the time between injury and reconstruction (8-10).

Systematic reviews tried to answer this question, however, because of the lack of high-level studies and considerable overlap in the definition of acute versus delayed reconstruction, it was difficult to come to conclusions (3, 11-13).

In more recent systematic reviews and meta-analysis, with the definitions for early and delayed reconstruction set at $<3$ weeks and $\geq 3$ weeks respectively, the authors found no differences regarding patient reported outcome measures (PROMs) and objective clinical assessments comparing early and delayed ACLR $(14,15)$.

The aim of this study was to compare clinical outcomes following ACLR performed in a very early phase post injury (maximum 2 weeks injury-surgery interval) with those of surgery performed at least 3 weeks post injury and to evaluate ROM difference at different follow-ups.

\section{METHODS}

All patients with a clinical and magnetic resonance imaging (MRI) diagnosis of ACL complete lesion, examined in the emergency department or the clinic of our hospital, were eligible for inclusion during a 1-year period beginning September 2018.

Inclusion criteria applied were unilateral primary ACL complete lesion diagnosed by positive Lachman and Pivot shift test and confirmed by MRI, age between 18 and 50 years, contralateral healthy knee, availability for early reconstruction and supervised rehabilitation protocol. Exclusion criteria were as follows: fibular collateral or posterolateral corner injury requiring surgery, medial collateral ligament (MCL) lesion greater than grade 1, posterior cruciate ligament (PCL) insufficiency, cartilage damage (grade 3 or 4 according to Outerbridge classification) (16); any prior surgery in the involved or contralateral knee, BMI $>30$, no radiographic signs of osteoarthritis, rheumatological disorders, associated malalignment (severe valgus $>7$ degree or varus knee deviation $>10$ degree), genu recurvatum $\left(>5^{\circ}\right.$ ), Beighton score 4 or more (hyperlaxity).

Patients meeting the eligibility criteria were prospectively enrolled in the study after informed consent was obtained. Patients who underwent surgery within 14 days of injury were assigned to group 1 (early ACLR) while patients who came to visit after two weeks from trauma or who chose to undergo late treatment were assigned to group 2 (delayed ACLR). In the delayed reconstruction group, ACLR was preceded by a preoperative rehabilitation protocol aimed to recover full range of motion and muscle tone and trophism. The study was approved by the local Ethical Committee (Nr. 0031/2018). All patients provided informed consent prior to participating in the study in accordance with the Declaration of Helsinki (1964). The study meets the ethical standards of the journal (17).

\section{Surgical technique}

An arthroscopically assisted anatomic single-bundle 2-incision technique using doubled semitendinosus and gracilis tendons (DGST) autografts was used for all patients. A standard tibial guide at $60-65^{\circ}$ was placed at the centre of the anatomic footprint of the ACL. The tunnel was drilled with the same diameter of the graft with an outside -in technique. On the femoral side, a 25 -mm-long bone socket was drilled with retrodrill (Flipcutter, Arthrex, Naples, FL) using a femoral guide at $100^{\circ}$ $110^{\circ}$ that was placed midway between resident ridge and over the top position (18).

The graft was passed through the tibial tunnel to the femoral socket and then fixed with the ACL TightRope (Arthrex, Naples, FL) on the femoral side and a bioabsorbable screw (Bio-Interference Screw; Arthrex, Naples, FL) on the tibia, sized $1 \mathrm{~mm}$ greater than the graft diameter and 28 or $35 \mathrm{~mm}$ in length.

\section{Post-operative protocol}

Pain relief after surgery was based on Paracetamol $1 \mathrm{~g}$ every 8 hours and NSAIDs (non steroidal anti-inflammatory drugs) as needed. In all patients the knee was placed in a full extension brace immediately after surgery, the first postoperative day partial weight-bearing was allowed as tolerated with crutches and the brace and daily isometric exercises were prescribed. The patients started physical therapy the day after surgery. After the hospital discharge, all the patients have done outpatient physiotherapy; they started progressive ROM exercises from the first postoperative day with the aim to maintain full extension and progressively recover flexion. At two weeks postoperatively, the patients come for a visit, medication of the surgical wound and suture removal. At this time the brace was unlocked to $0^{\circ}-90^{\circ}$. All patients were focused on reaching a full range of motion and full weight-bearing within 4 weeks. The brace and the crutches were abandoned at 4 weeks postoperatively. From the second month post-operatively, a heavier muscle-strengthening program was prescribed. Patients began gradual noncontact athletic activity and sport-specific training at 3 months. Return to full sports activities was allowed if the neuromuscular function had recovered, which usually occurs within 5-6 months. Timing and objectives of the postoperative physical therapy protocols are reported in table I. 
Table I. Rehabilitation protocol.

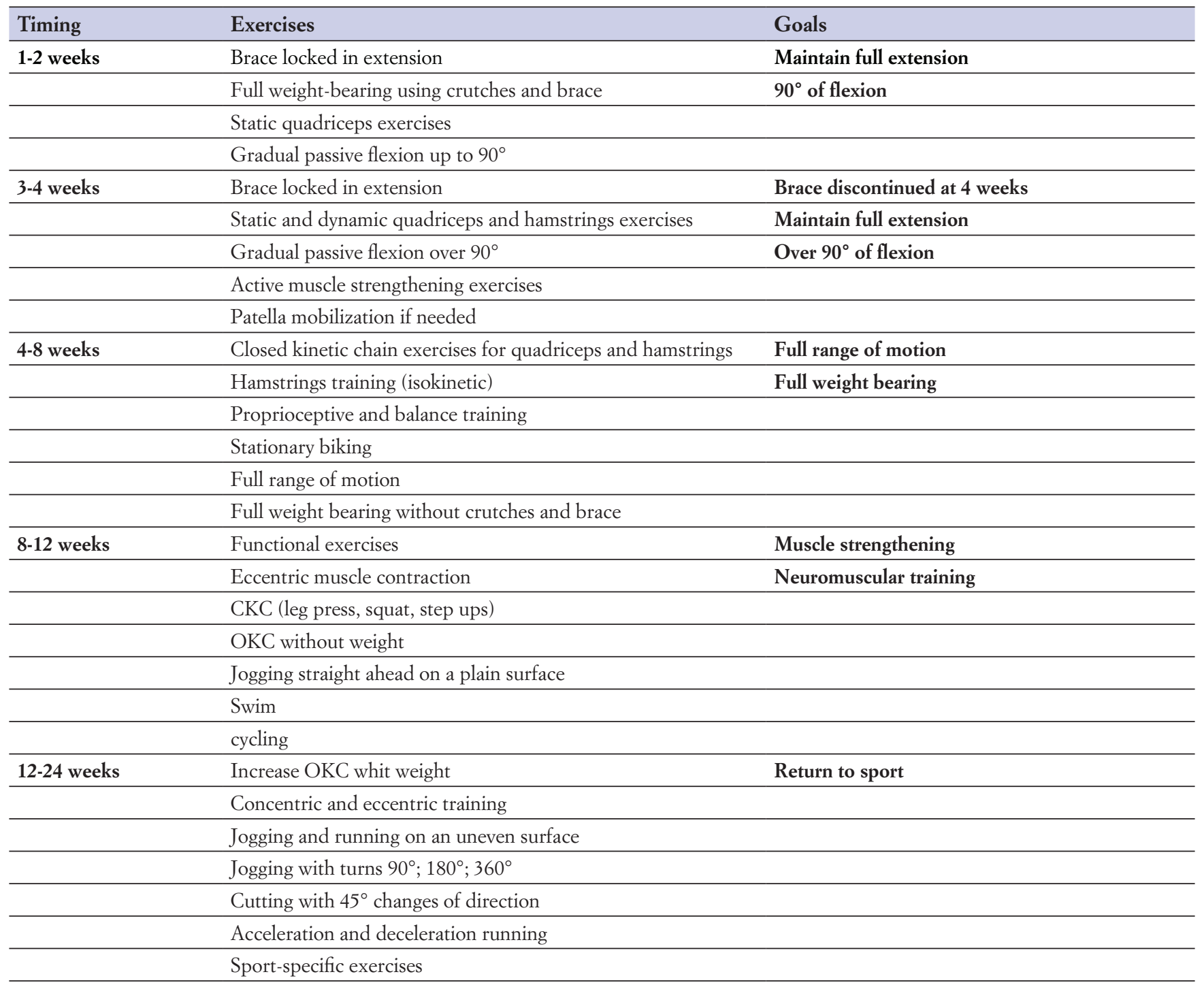

CKC: closed kinetic chain; OKC: open kinetic chain.

\section{Follow-up evaluation}

All patients were re-evaluated at 2, 4, 8, 12 and 24 weeks postoperatively by the same observer, a structured physiotherapist, who was independent and not involved in the initial surgery and blinded for the timing of the reconstruction. A standard physical knee examination with stability testing (Lachman and Pivot shift test) and passive ROM measurement, using a goniometer, were performed at each follow-up. Knee injury and osteoarthritis outcome score (KOOS) (19), Tegner Lysholm score (20), and International Knee Documentation Committee (IKDC 2000) (21) were obtained at final follow-up as well as manual maximum (MM) KT1000 evaluation (Medmetric,
San Diego, USA). Single leg hop test and thigh circumference measurement $(10 \mathrm{~cm}$ proximal to the proximal pole of the patella) were also performed at final follow up (22).

The Limb Symmetry Index (LSI) was calculated for all hop tests by dividing the result for the injured leg by that of the uninjured leg and multiplying by 100 (LSI = injuried leg / uninjuried leg $\times 100)(22)$.

\section{Statistical analysis}

Data were analysed with $\mathrm{IBM}^{\circledR}$ SPSS $^{\circledR}$ Statistics version 25 for MacOS, level of statistical significance was set at 0.05 (95\% interval of confidence). 
Descriptive statistics were conducted considering the nature of the criteria examined. For quantitative data, we included the number of observations, means and standard deviations. For qualitative data, we described the number of observed values and the percentage of patients by category. Nominal variables were tested by Pearson's $\chi 2$ test.

The distribution of values was assessed with the Kolmogorov-Smirnov test.

The ordinal variables normally distributed data were compared using Student's T-test while non-normally distributed data with the Mann-Whitney U Test.

We conducted a Relative Risk study to see if there was a risk of developing associated lesions over time injury-recon.

A sample size calculation was performed using, as the primary outcome variable, the ROM in flexion. With a mean difference of $5^{\circ}$ (corresponding to means of $130^{\circ}$ versus $125^{\circ}$ ), the study will have a power of $90 \%$ to yield a statistically significant result with $5 \%$ risk of a type-one error, with a proposed sample size of 42 patients, 21 for each group (23).

\section{RESULTS}

At the end of the selection process, 52 patients were enrolled in the study; 26 were assigned to the acute group and 26 to the delayed group. Demographic data are displayed in table II. Mean time between injury and reconstruction was 7 days in group 1 and 271 days in group 2 $(\mathrm{P}<0.001)$. Data regarding associated injuries are reported in table III. There were no differences in associated lesions between the two groups. The calculation of Relative Risk for the associated injuries between the two groups showed no differences.

There were no cases of arthrofibrosis in both groups. No significative difference was detected between groups regarding the range of motion; mean flexion and extension values at each follow up are shown in (table IV). At 8 weeks, $78 \%$ of patients in the group 1 (acute surgery) reached full ROM while $64 \%$ of patients reached complete ROM in the group 2 (delayed surgery). Full range of motion was achieved at 12 weeks in both groups (figure 1).

At final follow up no statistically significant difference was found between the two groups concerning IKDC, KOOS and Tegner Lysholm Knee Scoring Scale evaluations as well as knee laxity clinical examinations and manual maximum side to side difference at KT1000 measurements. (table V) Results regarding muscle circumference and functional strength at the single leg hop test (OLH) showed no significative difference between groups. The mean LSI value was 92.8 in group 1 and 92.4 in group 2 (table VI).

Table II. Demographic of the patient population.

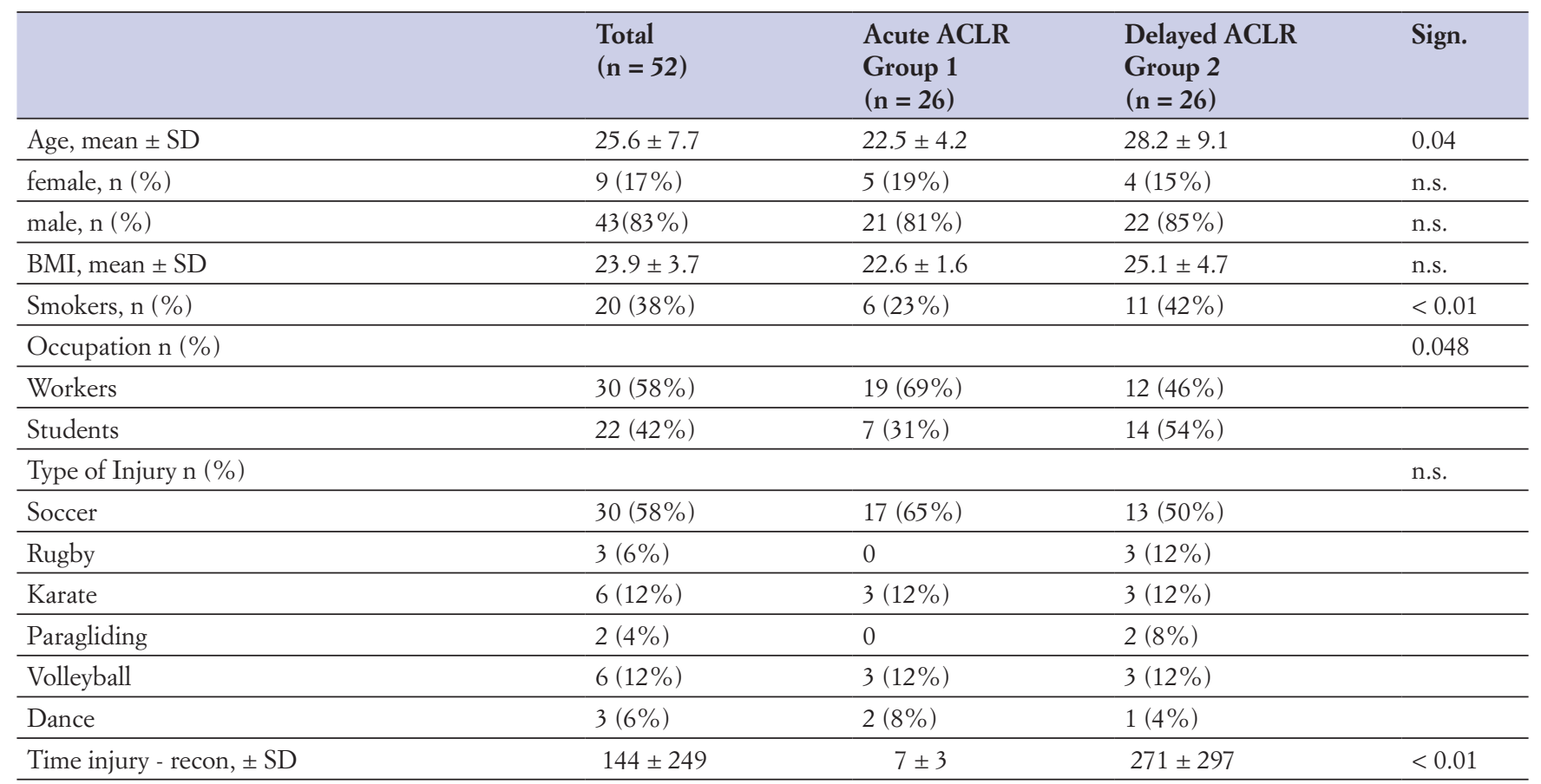

SD: standard deviation; ACLR: acute anterior cruciate reconstruction. Patient demographic are displayed as mean \pm standard deviation (SD), number and percentage. 
Table III. Associated injury.

\begin{tabular}{llll}
\hline & Acute ACLR $(\mathbf{n}=\mathbf{2 6})$ & Delayed ACLR $(\mathbf{n}=\mathbf{2 6})$ & Relative Risk \\
\hline MM bucket handle tear & 1 & 2 & 0.5 \\
\hline LM radial tear & 1 & 2 & 0.5 \\
\hline LMPHRT & 1 & 0 & n.s. \\
\hline Chondral injuries & 1 & 2 & 0.5 \\
\hline ACLR act
\end{tabular}

ACLR: acute anterior cruciate ligament reconstruction; MM: medial meniscus; LM: lateral meniscus; LMPHRT: lateral meniscus posterior horn root tears. The relative risk for LMPHRT was not calculable because there were no cases of lesion in the delayed ACLR group.

Table IV. Mean ROM Degree at 2-4-8-12-24 Weeks.

\begin{tabular}{|c|c|c|c|}
\hline & Acute ACLR $(n=26)$ & Delayed ACLR $(n=26)$ & Sign. \\
\hline \multicolumn{4}{|l|}{2 weeks } \\
\hline Extension \pm SD & $0.4 \pm 1.4$ & $0.3 \pm 2.8$ & n.s. \\
\hline \multicolumn{4}{|l|}{4 weeks } \\
\hline Extension $\pm \mathrm{SD}$ & $0.6 \pm 1.6$ & $0.9 \pm 1.8$ & n.s. \\
\hline Extension \pm SD & $0.4 \pm 1.5$ & $0 \pm 0$ & n.s. \\
\hline Flexion \pm SD & $127.3 \pm 9.0$ & $119.1 \pm 12.2$ & n.s. \\
\hline \multicolumn{4}{|l|}{12 weeks } \\
\hline Extension \pm SD & $0 \pm 0$ & $0 \pm 0$ & n.s. \\
\hline Flexion \pm SD & $130.0 \pm 0$ & $130.0 \pm 0$ & n.s. \\
\hline
\end{tabular}

ROM: range of motion; ACLR: acute anterior cruciate ligament reconstruction; SD: standard deviation. Data regarding ROM are reported with mean \pm SD.

Table V. Subjective and objective evaluations at 24 weeks

\begin{tabular}{llll}
\hline & Acute ACLR $(\mathbf{n}=\mathbf{2 6})$ & Delayed ACLR $(\mathbf{n}=\mathbf{2 6})$ & Sign. \\
\hline IKDC \pm SD & $98.7 \pm 3.3$ & $95.2 \pm 3.8$ & n.s. \\
\hline TEGNER LYSHOLM \pm SD & $100 \pm 0.0$ & $93.8 \pm 7.0$ & n.s. \\
\hline KOOS \pm SD & $99.0 \pm 1.8$ & $95.5 \pm 4.1$ & n.s. \\
\hline LACHMAN n $(\%)$ & & n.s. \\
\hline 0 & $25(100)$ & $26(100)$ & \\
\hline $1+$ & $0(0)$ & $0(0)$ & \\
\hline $2+$ & $0(0)$ & $0(0)$ & n.s. \\
\hline PIVOT SHIFT n $(\%)$ & & $23(89)$ & \\
\hline 0 & $23(92)$ & $3(11)$ & \\
\hline $1+$ & $2(8)$ & $0(0)$ & \\
\hline $2+$ & $0(0)$ & $0(0)$ & n.s.
\end{tabular}

SD: standard deviation; ACLR: anterior cruciate ligament reconstruction; IKDC:International Knee Documentation Committee; KOOS: Knee injury and Osteoarthritis Outcome Score. Data are displayed as mean \pm SD. 
Table VI. Functional strength.

\begin{tabular}{lllc}
\hline & Acute ACL-R & Delayed ACL-R & Sign. \\
\hline $\begin{array}{l}\text { OLH - injured leg (cm) } \\
\text { Mean (SD) }\end{array}$ & $91(25.5)$ & $82(20.6)$ & n.s. \\
\hline $\begin{array}{l}\text { OLH - uninjured leg (cm) } \\
\text { Mean (SD) }\end{array}$ & $\mathbf{9 9 . 9 ( 2 4 . 8 )}$ & $\mathbf{8 9 . 2 ( 1 8 . 8 )}$ & n.s. \\
\hline $\begin{array}{l}\text { OLH inj. - uninj. difference (cm) } \\
\text { Mean (SD) }\end{array}$ & $8.1(18.7)$ & $7.2(15)$ & n.s. \\
\hline $\begin{array}{l}\text { LSI (\%) } \\
\text { Mean (SD) }\end{array}$ & $\mathbf{9 2 . 8 ( 1 4 . 4 )}$ & $\mathbf{9 2 . 4 ( 1 8 . 2 )}$ & n.s. \\
\hline $\begin{array}{l}\text { Thighs circumference } \\
\text { inj. - uninj. difference (cm) } \\
\text { Mean (SD) }\end{array}$ & $2.1(1.3)$ & $2.9(2.3)$ & n.s. \\
\hline
\end{tabular}

SD: standard deviation; OLH: One Leg Hop; LSI: Limb Simmetry Index. Data are reported with mean \pm SD, differences are displayed in cm, thigh circumference was measured $10 \mathrm{~cm}$ above the patella.

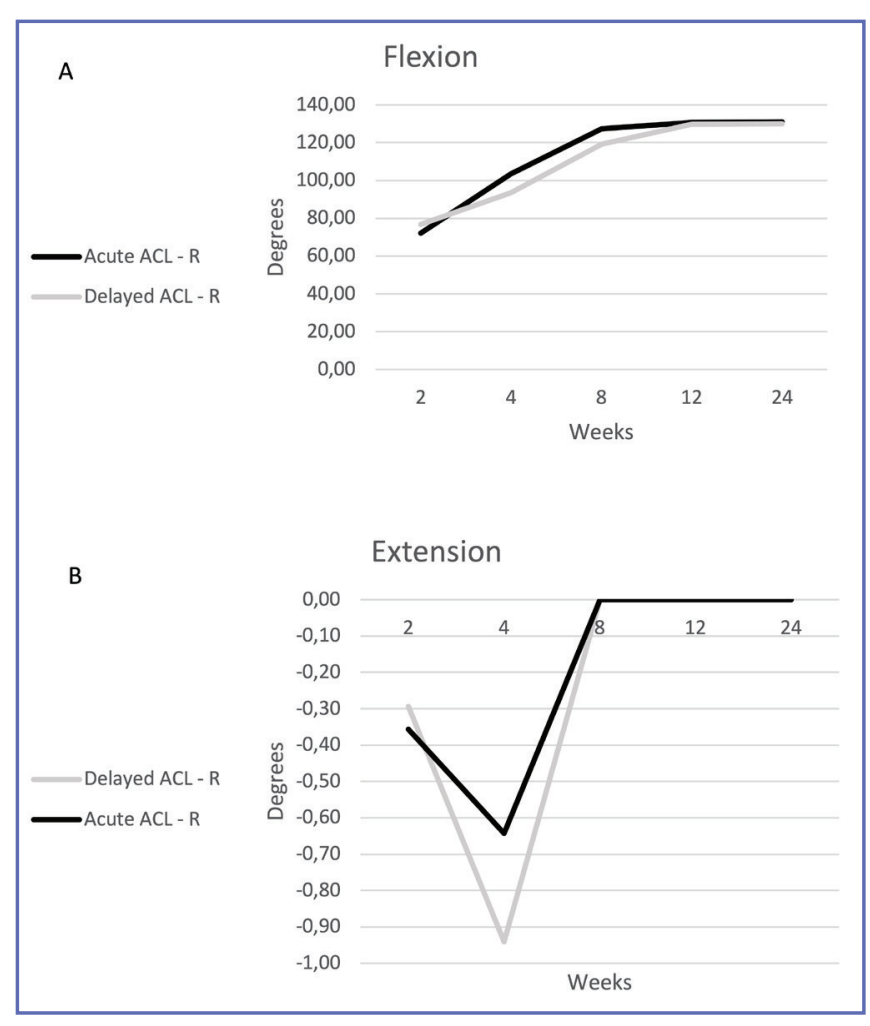

Figure 1. (A) Mean flexion at 2, 4, 8, 12 and 24 weeks; (B) mean extension deficit at 2, 4, 8,12 and 24 weeks.

\section{DISCUSSION}

The most important finding of this prospective study is that timing of ACLR does not influence postoperative ROM. Acute reconstruction ensures quick recovery with similar outcomes and functional strength at 6 months if compared to delayed reconstruction.
Arthrofibrosis of the knee is the most common post-operative complications of ACLR, with rates between $4 \%$ and $38 \%$ (24). Several definitions have been proposed for arthrofibrosis. According to Wasilewski et al. arthrofibrosis was a total loss of motion greater than $25 \%$ at 6 months postoperatively or knees requiring lysis of adhesions and manipulation (5). A study conducted by Shelbourne et al., classified arthrofibrosis of the knee in 4 types based on loss motion and patellar tightness and contracture compared with the other knee. Type 1 was $<10^{\circ}$ of extension loss and normal flexion. Type 2 was $>10^{\circ}$ of extension loss and normal flexion. Type 3 was $>10^{\circ}$ extension loss and $>25^{\circ}$ of flexion loss with patellar tightness and no patella infera. Type 4 was $>$ of $10^{\circ}$ extension loss, $30^{\circ}$ or more of flexion loss loss and objective patella infera with patellar tightness (25). A systematic review conducted by Ekthiari et al. analyzed 25 studies including 647 patients treated for arthrofibrosis after ACLR. They concluded that arthrofibrosis is poorly defined and outcomes measures varies widely (24).

The effect of the timing of ACLR on the development of post-operative arthrofibrosis has long been discussed. Older studies, supporting delayed reconstruction, reported a higher incidence of postoperative stiffness after acute ACLR, however most of these were retrospective studies that included patients with additional ligament injuries often operated with arthrotomy technique and more restrictive postoperative rehabilitation protocols $(4,5,26)$.

In 1990s Ferretti et al. published a medium-term follow-up study on a series of 55 cases of acute ACL hamstrings reconstruction, performed within ten days since the initial injury (27). Besides the overall good subjective and objective outcomes, the authors reported a lower rate of meniscectomies as compared with a comparable series of patients operated in a chronic phase. However, they also reported ten cases 
of manipulation under anaesthesia performed within three months since surgery in due to a delay in recovery of ROM as a result of an up to six weeks postoperative cast immobilization. In the present series, as a more accelerated rehabilitation was used this procedure has never been required.

The study by Shelbourne et al. was one of the first to recommend delaying an ACL reconstruction by at least 3 weeks (4). The authors reported a significantly increased incidence of arthrofibrosis in patients reconstructed within 8 days when compared with those reconstructed after 21 days. The authors also reported that patients treated between 8 and 21 days who underwent an aggressive rehabilitation program had better range of motion if compared to patients following conservative protocol. As a result, subsequent studies adopted a similar accelerated rehabilitation protocol (12, 13, 28). This, along with the use of modern surgery technique, may explain why recent studies reported no clinical difference between early versus chronic ACLR supporting our findings $(6,7,29)$.

Deabate et al. found no differences in terms of PROMs, risk of complications, ROM limitation, risk of re-tears, and residual laxity comparing early versus delayed ACLR (14). In the meta-analysis conducted by Ferguson et al., only statistically significant finding was for the Tegner activity scale, which demonstrated improved reported outcomes with early surgery (15).

No other findings reached significance, which was in keeping with other systematic reviews and meta-analysis reporting no differences in subjective and objective outcomes related to surgical timing of ACL reconstruction (3, 12-14). A systematic review and meta-analysis conducted by Smith et al. examined six papers including 370 ACLR. Early reconstructions were performed within a mean of 3 weeks from injury; delayed reconstructions were performed with a minimum of six weeks from injury. They found that there were no difference in clinical outcome between early and delayed reconstructions (13).

On KT-1000 arthrometric evaluation, Li et al. found evidence of greater stability with early surgery (30), conversely Raviraj et al. showed no differences between the groups as well as to clinical examination by Lachman, Pivot shift, and anterior draw testing (31).

Different studies reported other advantages of early surgery such as less muscle atrophy and less risk of exposing the knee to subsequent injuries associated with recurring instability such as meniscal and chondral injuries $(11,32)$. In our cohort of patients, we reported no difference regarding functional strength and associated lesions. Otherwise, Von Essen et al. showed superior outcome in the acute ACLR group regarding strength and how patients felt their knee functioning at 24 months (7).
It has been reported that the odds for cartilage lesion occurrence increases by nearly $1 \%$ for each month that elapses from injury to surgery, and that a lesion of the cartilage is associated with a 2 -fold increase in the risk of developing a meniscal tear (33).

In our study, we didn't detect a relevant greater number of associated lesions in the delayed ACLR group, but it might have existed if surgery had been delayed by months or years while in our group 2 (delayed surgery) the mean time injury surgery was 271 days ( 9 months).

Conteduca et al. as matter of fact, reported a statistically significant difference regarding the percentage of chondromalacia when comparing patients who waited less and more than 30 months (34). Moreover, in the work conducted by Ferretti et al. the incidence of meniscal tears was directly correlated with the time between the first injury and operation (8).

According to Bottoni et al. we believe that delayed surgery represents a "second hit" for the knee, on the contrary, if the surgery is performed proximate to the injury, the body has just one "trauma" from which to heal and only one rehabilitation program to deal with (29). This, along with a shorter injury-surgery time, can guarantee a faster and easier return to sport. Moreover, although in the absence of statistical significance, our results showed a trend towards a faster $\mathrm{ROM}$ recovery in a greater percentage of patients undergoing acute versus delayed ACLR.

This study has several strengths, one of which is the prospective design, all of the ACLRs were performed with the same surgical technique and our results were obtained with the same accelerated rehabilitation protocol that was minimally supervised. Furthermore, acute ACLRs have been performed in a very early phase with a mean time injury surgery of 7 days.

Potential limitations are the lack of randomization; the relative small number of patients and the relatively short postoperative follow-up; however, follow-up is not a major concern, as the primary outcome is return of range of motion.

\section{CONCLUSIONS}

Acute ACL reconstruction can be performed safely with no increased risk of arthrofibrosis and clinical outcomes are comparable to delayed ACLR.

Early reconstruction, if followed by an aggressive rehabilitation program, promotes a rapid recovery for motivated patients with only one rehabilitation protocol needed.

\section{CONFLICT OF INTERESTS}

The authors declare that they have no conflict of interests. 


\section{REFERENCES}

1. Moses B, Orchard J, Orchard J. Systematic review: Annual incidence of ACL injury and surgery in various populations. Res Sports Med 2012;20:157-79.

2. Iorio R, Iannotti F, Ponzo A, et al. Anterior cruciate ligament reconstruction in patients older than fifty years: a comparison with a younger age group. Int Orthop 2018;42:1043-49.

3. Lee YS, Lee OS, Lee SH, Hui TS. Effect of the Timing of Anterior Cruciate Ligament Reconstruction on Clinical and Stability Outcomes: A Systematic Review and Meta-analysis. Arthroscopy 2018;34:592-602.

4. Shelbourne KD, Wilckens JH, Mollabashy A, DeCarlo M. Arthrofibrosis in acute anterior cruciate ligament reconstruction. The effect of timing of reconstruction and rehabilitation. Am J Sports Med 1991;19:332-6.

5. Wasilewski SA, Covall DJ, Cohen S. Effect of surgical timing on recovery and associated injuries after anterior cruciate ligament reconstruction. Am J Sports Med 1993;21:338-42.

6. Eriksson $\mathrm{K}$, von Essen C, Jonhagen S, Barenius B. No risk of arthrofibrosis after acute anterior cruciate ligament reconstruction. Knee Surg Sports Traumatol Arthrosc 2018;26:2875-82.

7. von Essen C, Eriksson K, Barenius B. Acute ACL reconstruction shows superior clinical results and can be performed safely without an increased risk of developing arthrofibrosis. Knee Surg Sports Traumatol Arthrosc 2020;28:2036-43.

8. Ferretti A, Conteduca F, De Carli A, Fontana M, Mariani PP. Osteoarthritis of the knee after ACL reconstruction. Int Orthop 1991;15:367-71.

9. Krutsch W, Zellner J, Baumann F, Pfeifer C, Nerlich M, Angele P. Timing of anterior cruciate ligament reconstruction within the first year after trauma and its influence on treatment of cartilage and meniscus pathology. Knee Surg Sports Traumatol Arthrosc 2017;25:418-25.

10. Mather RC 3rd, Hettrich CM, Dunn WR, et al. Cost-Effectiveness Analysis of Early Reconstruction Versus Rehabilitation and Delayed Reconstruction for Anterior Cruciate Ligament Tears. Am J Sports Med 2014;42:1583-91.

11. Andernord D, Karlsson J, Musahl V, Bhandari M, Fu FH, Samuelsson K. Timing of surgery of the anterior cruciate ligament. Arthroscopy 2013;29:1863-71.

12. Kwok CS, Harrison T, Servant C. The optimal timing for anterior cruciate ligament reconstruction with respect to the risk of postoperative stiffness. Arthroscopy 2013;29:556-65.

13. Smith TO, Davies L, Hing CB. Early versus delayed surgery for anterior cruciate ligament reconstruction: a systematic review and meta-analysis. Knee Surg Sports Traumatol Arthrosc 2010;18:304-11.

14. Deabate L, Previtali D, Grassi A, Filardo G, Candrian C, Delcogliano M. Anterior Cruciate Ligament Reconstruction Within 3 Weeks Does Not Increase Stiffness and Complications Compared With Delayed Reconstruction: A Meta-analysis of Randomized Controlled Trials. Am J Sports Med 2020;48:1263-72.

15. Ferguson D, Palmer A, Khan S, Oduoza U, Atkinson H. Early or delayed anterior cruciate ligament reconstruction: Is one superior? A systematic review and meta-analysis. Eur J Orthop Surg Traumatol 2019;29:1277-89.
16. Outerbridge RE. The etiology of chondromalacia patellae. J Bone Joint Surg Br 1961;43-B:752-7.

17. Padulo J, Oliva F, Frizziero A, Maffulli N. Basic principles and recommendations in clinical and field Science Research: 2018 update. Muscles Ligaments Tendons J 2018;8(3):305-7.

18. Purnell ML, Larson AI, Clancy W. Anterior cruciate ligament insertions on the tibia and femur and their relationships to critical bony landmarks using high-resolution volume-rendering computed tomography. Am J Sports Med 2008;36:2083-90.

19. Roos EM, Roos HP, Lohmander LS, Ekdahl C, Beynnon BD. Knee Injury and Osteoarthritis Outcome Score (KOOS)--development of a self-administered outcome measure. J Orthop Sports Phys Ther 1998;28:88-96.

20. Tegner Y, Lysholm J. Rating systems in the evaluation of knee ligament injuries. Clin Orthop Relat Res 1985;(198):43-9.

21. Hefti F, Muller W, Jakob RP, Staubli HU. Evaluation of knee ligament injuries with the IKDC form. Knee Surg Sports Traumatol Arthrosc 1993;1:226-34.

22. Barber SD, Noyes FR, Mangine RE, McCloskey JW, Hartman W. Quantitative assessment of functional limitations in normal and anterior cruciate ligament-deficient knees. Clin Orthop Relat Res 1990;(255):204-14.

23. Julious SA. Sample sizes for clinical trials with normal data. Stat Med 2004;23:1921-86

24. Ekhtiari S, Horner NS, de Sa D, et al. Arthrofibrosis after ACL reconstruction is best treated in a step-wise approach with early recognition and intervention: a systematic review. Knee Surg Sports Traumatol Arthrosc 2017;25:3929-37.

25. Shelbourne KD, Patel DV, Martini DJ. Classification and management of arthrofibrosis of the knee after anterior cruciate ligament reconstruction. Am J Sports Med 1996;24:857-62.

26. Mohtadi NG, Webster-Bogaert S, Fowler PJ. Limitation of motion following anterior cruciate ligament reconstruction. A case-control study. Am J Sports Med 1991;19:620-4; discussion 624-5.

27. Ferretti A, Conteduca F, De Carli A, Fontana M, Mariani PP. Results of reconstruction of the anterior cruciate ligament with the tendons of semitendinosus and gracilis in acute capsulo-ligamentous lesions of the knee. Ital J Orthop Traumatol 1990;16:452-8.

28. Beynnon BD, Uh BS, Johnson RJ, et al. Rehabilitation after anterior cruciate ligament reconstruction: a prospective, randomized, double-blind comparison of programs administered over 2 different time intervals. Am J Sports Med 2005;33:347-59.

29. Bottoni CR, Liddell TR, Trainor TJ, Freccero DM, Lindell KK. Postoperative range of motion following anterior cruciate ligament reconstruction using autograft hamstrings: a prospective, randomized clinical trial of early versus delayed reconstructions. Am J Sports Med 2008;36:656-62.

30. Li B, Bai L, Fu Y, Wang G, He M, Wang J. Effect of timing of surgery in partially injured ACLs. Orthopedics 2012;35:408-12.

31. Raviraj A, Anand A, Kodikal G, Chandrashekar M, Pai S. A comparison of early and delayed arthroscopically-assisted reconstruction of the anterior cruciate ligament using hamstring autograft. J Bone Joint Surg Br 2010;92:521-6.

32. Marcacci M, Zaffagnini S, Iacono F, Neri MP, Petitto A. Early versus late reconstruction for anterior cruciate liga- 
ment rupture. Results after five years of followup. Am J Sports Med 1995;23:690-3.

33. Granan LP, Bahr R, Lie SA, Engebretsen L. Timing of anterior cruciate ligament reconstructive surgery and risk of cartilage lesions and meniscal tears: a cohort study based on the Norwegian National Knee Ligament Registry. Am J Sports Med 2009;37:955-61.

34. Conteduca F, Ferretti A, Mariani PP, Puddu G, Perugia L. Chondromalacia and chronic anterior instabilities of the knee. Am J Sports Med 1991;19:119-23. 